\title{
The relaxed structure of intrinsic dislocation networks in semicoherent interfaces: predictions from anisotropic elasticity theory and comparison with atomistic simulations
}

\author{
A. Vattréa, ${ }^{a, *}$ N. Abdolrahim ${ }^{\mathrm{b}}$, S.S. Navale ${ }^{\mathrm{c}}$, M.J. Demkowicz ${ }^{\mathrm{c}}$ \\ ${ }^{a}$ CEA, DAM, DIF, F-91297 Arpajon, France \\ ${ }^{b}$ Department of Mechanical Engineering, University of Rochester, NY, 14627 \\ ${ }^{c}$ Department of Materials Science and Engineering, Texas A\&M University, College \\ Station TX, 77843
}

\begin{abstract}
We investigate the effect of nodal relaxation on the structure and energy of interfacial dislocation networks predicted by a dislocation-based simulation method. To assess the accuracy of these predictions, we compare them to corresponding atomistic simulations. Two types of interfaces are investigated: pure twist grain boundaries along $\{110\}$-type planes in niobium as well as heterophase interfaces between $\{111\}$-type planes in silver and $\{110\}$ type interfaces in vanadium. We find that dislocation core energies play a major role in the former, leading to significant discrepancies between the dislocation-based model and atomistic simulations. By contrast, core contributions do not appear to be significant in the latter, giving rise to excellent agreement between the atomistic and dislocation-based models.
\end{abstract}

\footnotetext{
*Email address: aurelien.vattre@cea.fr
} 


\section{Introduction}

The internal structure of solid-state interfaces plays a decisive role in determining their properties. For example, disparities in the mechanical behavior of metal nanocomposites of unlike compositions have been attributed to differences in the internal structure of the heterophase interfaces found

within them $[1,2]$. Similarly, distributions of interfacial precipitates $[3,4]$ and the capacities of interfaces to absorb point defects $[5,6,7]$ have been linked directly to interface structures. With increasing interest in elucidating interface structure-property relations $[2,8]$, there is a growing need for models capable of predicting interface structures accurately and efficiently. We present a comparison of structure predictions for semicoherent interfaces made by two different methods: atomistic modeling and an elasticity-based reduced order model that accounts for the relaxation of interface dislocation networks $[9,10]$. Two types of model interface are considered: twist grain boundaries (t-GBs) on $\{110\}$ planes in niobium $(\mathrm{Nb})$ and heterophase interfaces between silver (Ag) and vanadium (V).

In metals, atomistic simulations provide the most direct means for investigating interface structure [8]. They model the positions of all atoms in the vicinity of an interface explicitly and do not rely on any prior understanding of interface structure. However, they are relatively resource-intensive and therefore do not lend themselves to rapid investigation of numerous interfaces [11]. An alternative approach is to recognize that predicting interface properties need not require modeling interface structure with atomic-level resolution. If the features of interface structure that govern interface behavior are known, then reduced order models (ROMs)-ones that only account 
for essential features and not for all atoms-may be constructed. Several classes of ROMs for interface structure are currently under investigation $[12,13,14,15]$. Here, we investigate dislocation-based ROMs that account for the relaxation of dislocation networks and their accuracy at predicting the structures and relative energies of $\mathrm{Nb}$ t-GBs and $\mathrm{Ag} / \mathrm{V}$ interfaces. Our findings provide insight into the strengths and weaknesses of these models and provide a perspective on avenues for their continued development.

Dislocation-based ROMs for interfaces are based on the fact that any difference in orientation (i.e., misorientation) or lattice strain (misfit) between two adjacent crystals may be generated by a suitably constructed network of dislocations confined to an interface plane of any spatial orientation $[16,17,18]$. In other words, the crystallographic character of any interface may be represented analytically by a two-dimensional dislocation network. For some interfaces, especially ones involving high misoriention angles [19], dislocation networks constitute poor models for the actual atomic-level interface structure. Nevertheless, some modeling studies have found [11, 20] that the range of interfaces whose atomic structure is accurately represented by dislocation networks is wider than originally anticipated [21]. In addition, dislocation models have yielded remarkably accurate predictions of the physical properties of several interfaces, including their energies [11, 21], interactions with point defects $[22,23]$, and mechanical properties [24, 25, 26]. Thus, dislocation-based ROMs for interface structure and properties remain under active development.

Dislocation network structures representing an interface of given crystallographic character may be obtained using the quantized Frank-Bilby equa- 
tion (qFBE) $[16,17]$. Indeed, for any given interface, the $\mathrm{qFBE}$ generally yields a large number of dislocation networks, each consistent with the crystallography of the interface under consideration, but with widely differing dislocation densities and characters [18]. To determine which of these networks is likeliest to represent the true structure of the interface, we compute the energy of each network and identify the one with the lowest energy as the best candidate $[11,27]$.

Our energy calculation is based on linear anisotropic elasticity theory. In previous studies $[11,22,27,28,29]$, we used this approach to compute the elastic energies of interface dislocation networks comprised of two overlapping arrays of straight, periodically-spaced dislocations. Thus, in these calculations, all intersections between dislocations occur at four-fold junctions. However, in many cases, such four-fold junctions split up into pairs of three-fold junctions connected by a new dislocation segment [30], causing the dislocation network to relax from a structure consisting of a periodic tiling of parallelograms to one composed of a periodic tiling of distorted hexagons $[31,32]$. Recently, it has become possible to model such relaxations using anisotropic elasticity theory $[9,10]$. The goals of the present study are therefore to a) model the elastic relaxation of dislocation networks for selected interfaces, b) validate this calculation by comparing the structures and energies of the relaxed networks to corresponding atomistic simulations, and c) assess the need for incorporating elastic relaxations into future simulations of interface dislocation networks. 


\section{Model interfaces}

We select model interfaces for our investigation according to the following criteria:

a) The structure of the interface is describable as a dislocation network. The present study is concerned with dislocation-based models of interface structure. Thus, interfaces to which these models do not apply are not suitable.

b) This dislocation network undergoes a relaxation via the dissociation of four-fold junctions into three-fold junctions. Some interfacial dislocation networks are not suitable for our study because they contain stable four-fold junctions that do not undergo any relaxation. Examples are pure twist boundaries on $\{100\}$-type planes in face centered-cubic (fcc) and $\mathrm{L} 1_{0}$-ordered crystals $[22,29]$.

c) The interface dislocation network is initially periodic and remains so as it relaxes. Moreover, the dislocations in the network do not dissociate into partials. These choices are necessitated by current limitations in modeling capabilities $[9,10]$. The requirement of periodicity is met by selecting special interfaces that may be modeled by two overlapping sets of misfit dislocations (whereas general interfaces involve three overlapping dislocation sets [33]). The requirement of no dissociation excludes from consideration grain boundaries in low stacking fault energy materials.

d) The final structure of the relaxed interface is not the outcome of any inherent symmetry that the interface possesses. For example, while 
twist boundaries on $\{111\}$ planes in aluminum meet all the foregoing conditions, they are excluded from consideration because the relaxed dislocation structure in these interfaces has the same $p 6 m$ symmetry as the underlying, unrelaxed dichromatic pattern $[34,35]$. In our view, such a symmetry-driven relaxation does not constitute a stringent test of the elasticity-based relaxation model we are examining in the present study.

e) Differences between the relaxed and unrelaxed dislocation network must be discernable in atomistic simulations. Thus, the dislocations should not be so closely spaced that they are difficult to distinguish yet not so far apart that they would require very large atomistic models. We meet this criterion through judicious selection of the interface crystallographic character (misorientation, misfit, and plane orientation).

All of the foregoing criteria are met by the two classes of model interfaces selected for our study: low-angle twist grain boundaries on $\{110\}$-type planes in niobium ( $\mathrm{Nb}$ t-GBs) as well as heterophase interfaces between $\{111\}$ type planes of silver and $\{110\}$-type planes of vanadium (Ag/V interfaces). Figures (1.a) and (1.b) illustrate the crystallography of these two interfaces, respectively. For both interface types, we consider a series of structures of varying twist angle, $\theta$, defined as shown in Fig. (1). We investigate $0^{\circ} \leq \theta \leq$ $10^{\circ}$ for both interface types. When $\theta=0^{\circ}$, the $\mathrm{Nb}$ t-GB reduces to a perfect single crystal while the $\mathrm{Ag} / \mathrm{V}$ interface is in the Nishiyama-Wasserman (NW) orientation relation [36, 37], where $\langle 110\rangle_{\mathrm{fcc}}$ and $\langle 100\rangle_{\mathrm{bcc}}$ are parallel within the interface plane (fcc: face centered-cubic; bcc: body centered-cubic).

$\mathrm{Ag} / \mathrm{V}$ interfaces formed in magnetron sputtered multilayers have been 

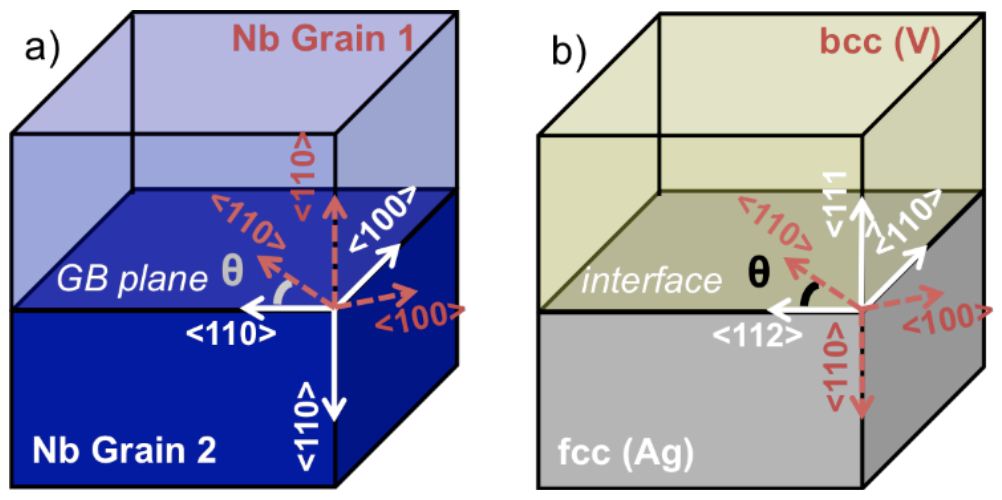

Figure 1: The crystallographic character of (a) low-angle twist grain boundaries on $\{110\}$ type planes in niobium ( $\mathrm{Nb}$ t-GBs) and (b) heterophase interfaces between $\{111\}$-type planes of silver and $\{110\}$-type planes of vanadium $(\mathrm{Ag} / \mathrm{V}$ interfaces). For both types of interfaces, we consider a series of structures with different twist angles, $\theta$.

characterized extensively [38]. They are observed in a variety of orientation relations (ORs) and with a wide range of interface planes. Among the structures reported are $\mathrm{Ag} / \mathrm{V}$ interfaces in the Kurdjumov-Sachs and NishiyamaWasserman ORs, both along Ag $\{111\}$ and V $\{110\}$ planes. Such interfaces are members of the class illustrated in Fig. (1.b). They have been previously modeled using elasticity theory, albeit without accounting for network relaxations [11, 39], as well us using classical potential [39]. Comparisons with atomistic simulations revealed discrepancies that were hypothesized to arise from nodal reconstructions of the kind investigated here.

No experimental investigations of $\mathrm{Nb}\{110\}$ t-GBs have been reported. Nevertheless, these interfaces were previously investigated by atomistic simulations [40], by anisotropic linear elasticity theory, with network relaxations accounted for [10], and most recently using phase field models [41]. However, no quantitative comparison between structures predicted by ROMs and atomistic modeling has been previously conducted. 


\section{Simulation methods}

\subsection{Atomistic modeling}

Figures (2.a) and (2.b) show the simulation cells we use to model $\mathrm{Nb}$ t-GBs and $\mathrm{Ag} / \mathrm{V}$ interfaces, respectively. In both cases, the interface plane lies in the $(\boldsymbol{x}, \boldsymbol{y})$ plane and twist angles $\theta$ are applied by rotating the top (V or G1) crystal about the $z$ axis. $\mathrm{Nb}$ t-GBs are constructed within a sphere of radius $R_{0}$ while $\mathrm{Ag} / \mathrm{V}$ interfaces are contained within a cylinder of radius $R_{0}$ and height $2 h$. Both models terminate with free surfaces in all directions. The models are subdivided into concentratic shells, as illustrated in the cross-sections shown in Fig. (2.a) and (2.b).

We use embedded atom method (EAM) potentials [42] to model atomic interactions in both $\mathrm{Nb}$ [43] and $\mathrm{Ag} / \mathrm{V}$ [44]. To relax as-constructed interface models, neighboring metal blocks are first translated rigidly with respect to each other in directions parallel to the interface. The lowest energy configuration from such translations is subsequently relaxed by conjugate gradient potential energy minimization (PEM) [45]. Only atoms within the radius $R_{s}$, shown in Fig. (2.c) and (2.d), are allowed to move. The positions of all atoms in the outer shell $\left(R_{s} \leq r \leq R_{0}\right)$ are kept fixed throughout the relaxation. Because the long-range stresses generated by interface misfit dislocations are perfectly cancelled by the interface's coherency stresses [27], the shape of the relaxed dislocation network is determined solely by short-range elastic interactions between nearby segments. Therefore, the outer surfaces of our atomistic models only influence the shape of the dislocation network within a region whose dimensions are of the same order as the periodic repeat unit of the network. PEM is considered to have converged when the two-norm of 
a)

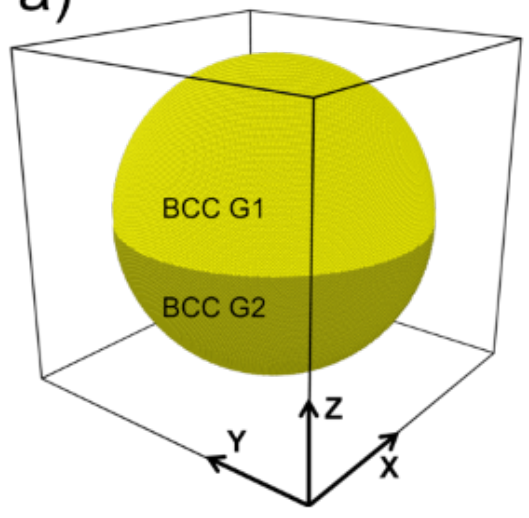

c)

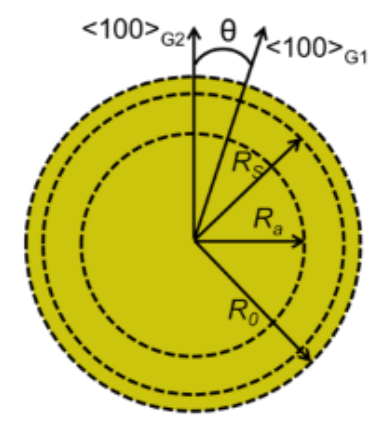

b)

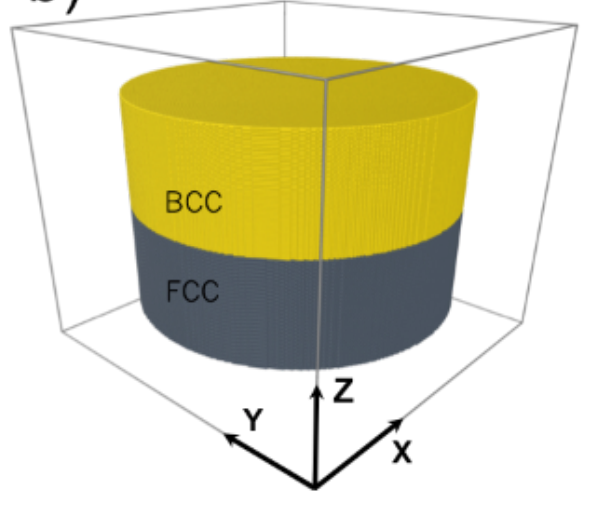

d)

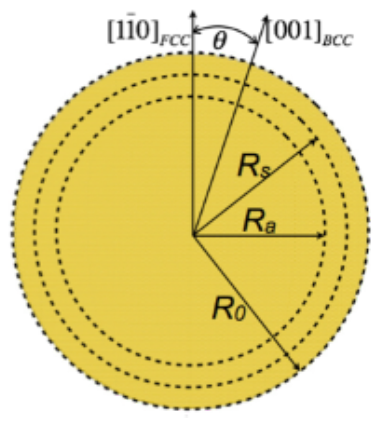

Figure 2: Simulation cells (a) Nb t-GB and (b) Ag/V interface models. The models consist of three concentric shells, as illustrated in (c) and (d) for $\mathrm{Nb}$ t-GB and $\mathrm{Ag} / \mathrm{V}$, respectively.

the force on every atom allowed to move is less than $0.1 \mathrm{pN}$. When relaxing $\mathrm{Nb}$ t-GBs, we additionally perform a 40 ps anneal at $300 \mathrm{~K}$ anneal followed by PEM relaxation after the initial rigid body translation and PEM steps.

To ensure that free surface and edge effects are minimized, we compute interface energies considering only atoms that lie within a region of radius $R_{a}$. For $\mathrm{Nb} \mathrm{t}-\mathrm{GB}$, we use $R_{s}=R_{0}-1 \mathrm{~nm}$, and $R_{a}=R_{0}-3 \mathrm{~nm}$. We select the model size so that the region with $R_{a}$ contains a representative area of interface with several unit cells of the interface's dislocation network. In the case of $\mathrm{Nb}$ t-GBs, we use $R_{0}=20 \mathrm{~nm}$ when $\theta>1^{\circ}$ and $R_{0}=40 \mathrm{~nm}$ 
otherwise. For the $\mathrm{Ag} / \mathrm{V}$ interface, we used $R_{0}=27 \mathrm{~nm}, R_{s}=R_{0}-2 \mathrm{~nm}$, $R_{a}=R_{0}-5 \mathrm{~nm}$, and $h=10 \mathrm{~nm}$. Increasing $R_{0}, R_{0}-R_{s}, R_{0}-R_{a}$, or $h$ beyond these values does not change the interface structures or energies reported below. By varying the radii over which interface energies are averaged, we estimate that, in our calculations, $\mathrm{Nb}$ t-GB energies are converged to within $\sim 20 \mathrm{~mJ} / \mathrm{m}^{2}$ and $\mathrm{Ag} / \mathrm{V}$ interfaces to within $\sim 5 \mathrm{~mJ} / \mathrm{m}^{2}$.

We compute $\mathrm{Nb}$ t-GB energies using

$$
\gamma_{\mathrm{GB}}=\frac{\left(E_{f}-E_{i}\right)}{\pi R_{a}^{2}}
$$

where $E_{f}$ is the sum of energies of all atoms in a cylindrical region of radius $R_{a}$ and height $2 h=10 \mathrm{~nm}$ in the relaxed interface model and $E_{i}$ is the product of cohesive energy a $\mathrm{Nb}$ atom in perfect crystal and total number of $\mathrm{Nb}$ atoms in the aforementioned cylindrical region. The interface energy for $\mathrm{Ag} / \mathrm{V}$ models is computed as

$$
\gamma_{\mathrm{fcc}-\mathrm{bcc}}=\frac{\left(E_{f}-E_{i}\right)}{\pi R_{a}^{2}}-\gamma_{(111)_{\mathrm{fcc}}}-\gamma_{(100)_{\mathrm{bcc}}} .
$$

Here, $E_{i}$ is the sum of the energies of all the atoms in the cylinder of radius $R_{a}$ when the fcc and bcc cylinder blocks are far enough from each other $(2.5 \mathrm{~nm}$ in our case) that there are no interactions between fcc and bcc free surfaces while $E_{f}$ is the sum of the energies of all atoms in the cylinder of radius $R_{a}$ after relaxation. Surface energies $\gamma_{(111)_{\mathrm{fcc}}}$ and $\gamma_{(100)_{\mathrm{bcc}}}$ are found in a separate calculation by dividing the difference in energies of atomic blocks with and without free surfaces by the area of the surfaces using the same interatomic potentials as the ones used in constructing heterointerfaces.

Interface dislocation cores were identified using local atomic energies. All atomistic calculations were conducted using the LAMMPS software package 
[46]. Visualizations were performed using AtomEye [47].

\subsection{Anisotropic, linear-elastic model}

The ROM used in the present work augments the geometrical constraints encapsulated in the qFBE with strain energy calculations via the Stroh formalism of anisotropic elasticity [48, 49]. In this approach, candidate dislocation-based representations of an interface of specified crystallographic character are enumerated from the qFBE [18]. For any such representation, a unique, coherent, single crystal reference state may be obtained by requiring mutual cancellation of coherency stresses with the long-range stresses generated by interface dislocations [27]. The Burgers vectors of the interface dislocations are taken to be irreducible translation symmetry vectors of this reference crystal.

Although numerous dislocation-based representations may be constructed for any interface based on the qFBE, in the present work we focus only on those with lowest elastic strain energy. For interfaces such as those investigated here-namely, ones represented by two sets of infinitely long, straight, parallel, and uniformly spaced dislocations-elastic strain energies may be computed using the method described in Ref. [27]. This approach takes advantage of the translational symmetry of such interfaces, representing the misfit-induced displacement field around them using Fourier series. For this calculation, it is essential that the net far-field stresses be zero, reinforcing

the importance of selecting the correct coherent reference state [29]. As in previous studies, all of our elastic energy calculations impose a dislocation core cutoff radius equal to one quarter of the dislocation Burgers vector magnitude. In section 4.2), we also investigate the effect of increasing the cutoff 
radius to twice the Burgers vector magnitude.

Unlike previous studies, the present work considers relaxations of dislocation networks via splitting of four-fold junctions into pairs of three-fold junctions. The three-fold junctions are connected by a dislocation segment formed through a reaction of the dislocations that intersect at the initial, four-fold junction. This relaxation preserves the translational symmetry of the dislocation pattern, but modifies the shape of the unit cell from a general parallelogram ("lozenge") to a distorted hexagon. Because the dislocation pattern remains translationally symmetric, the elastic displacements it generates are still conveniently represented using Fourier series. The corresponding elastic strain energy may still be computed using the Stroh formalism, as described in Ref. [9].

The relaxation described above may be fully parameterized by two degrees of freedom corresponding to the relative position of the three-fold junctions in the interface plane. To determine whether a relaxation takes place, the self and interaction contributions to the total elastic strain energy are computed. For all dislocation configurations with attractive interaction energies, fullyrelaxed patterns are found by exploring the energy landscape defined by relative nodal positions. Minimum-energy paths from the initial to the fullrelaxed states are obtained using the nudged elastic band method [9].

\section{Comparison of ROM predictions with atomistic models}

\section{1. $N b\{110\} t-G B s$}

Figure (3) compares the energy of $\mathrm{Nb}$ t-GBs computed from atomistic models with values obtained using our dislocation-based ROM, the latter using two different core cutoff radii. Both atomistics and the ROM reveal sim- 
ilar trends, with energies increasing monotonically as a function of $\theta$ within the range of twist angles investigated. Comparison of ROM results before and after relaxation of the dislocation network shows that this step in the calculation yields a relatively modest reduction in elastic energies. For example, for $\theta=2^{\circ}$, the reduction is approximately $8 \%$ of the initial energy. Energies computed from atomistic models are higher than those obtained from the ROM. This difference is due to dislocation core energies, which are inherently captured in the atomistic calculation, but are not accounted for in the ROM. The larger the core cutoff, the lower the energy computed by the ROM. Interestingly, regardless of the cutoff radius, the ROM values are smaller than the atomistic ones by an apparently $\theta$-independent factor, consistent with both the elastic and core energies scaling in proportion to the total length of dislocation segments in the network, to a first approximation.

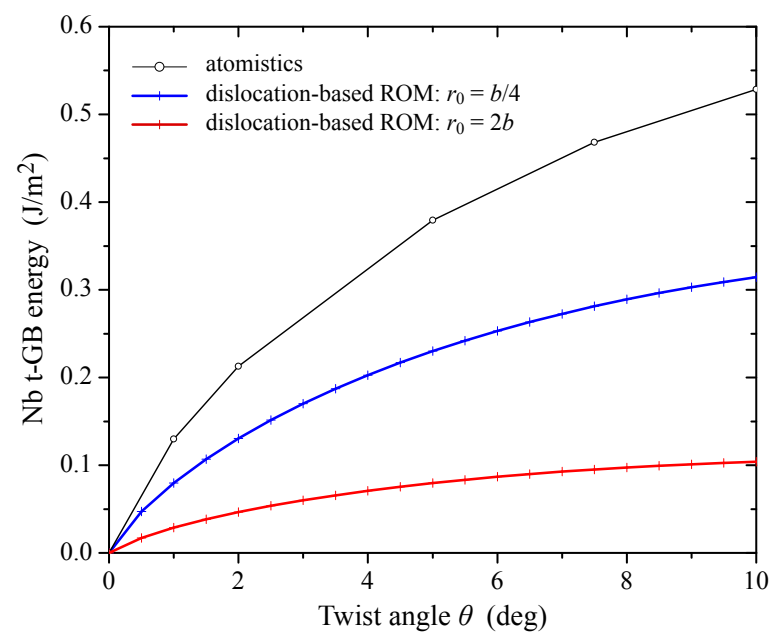

Figure 3: Nb-t GB energies computed as a function of $\theta$ using our dislocation-based ROM and atomistic modeling.

Figure (4) compares the structure of Nb t-GB dislocation networks deter- 
mined from atomistic modeling to ones found with our ROM, using $\theta=2^{\circ}$ as an example. Other twist angles give rise to qualitatively similar structures. The atomistic structure in Fig. (4.a) consists of a 2-D tiling of hexagonal regions separated by a connected network of misfit dislocation segments of predominantly screw character. Two types of segments are present: ones with $\frac{1}{2}\langle 111\rangle$-type Burgers vectors as well as ones with $\langle 100\rangle$-type Burgers vectors. As shown in Fig. (4.a), the former are approximately twice as long as the latter. Consistent with previous studies in bcc Nb [40] and iron [50], both segment types have compact cores of atomic-scale dimensions. The hexagonal regions making up the t-GB are symmetric with respect to reflections about mirror lines parallel and perpendicular to the shorter segments (with $\langle 100\rangle$-type Burgers vectors).
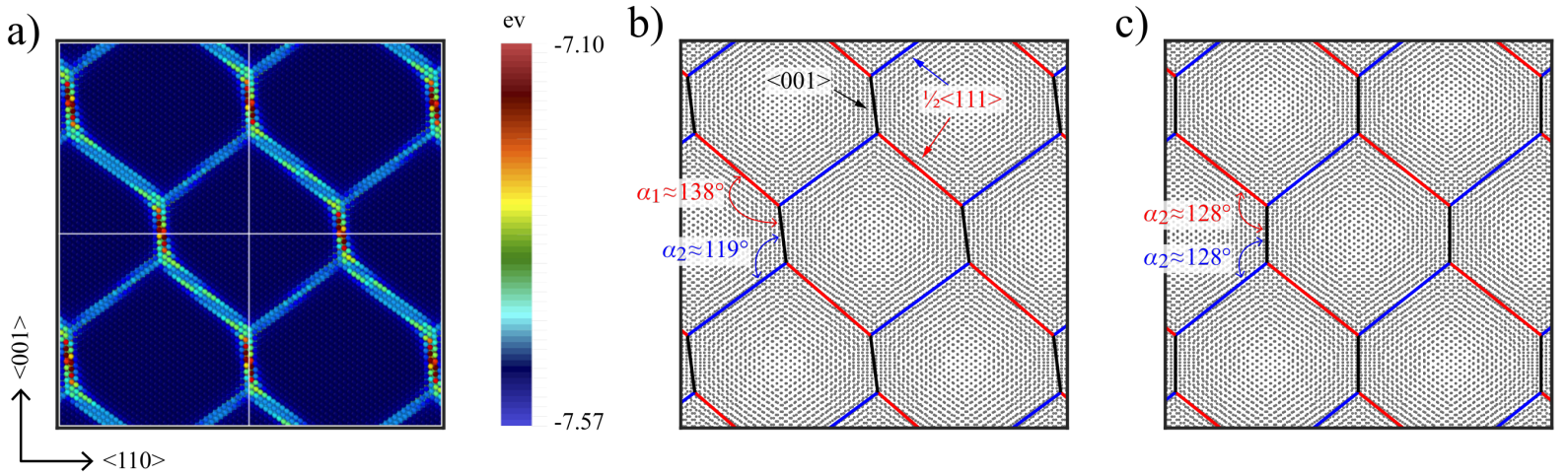

Figure 4: Dislocation networks in Nb t-GBs obtained by (a) atomistic modeling and the dislocation-based ROM with core cutoff radii of (b) and one quarter of the Burgers vector magnitude and (c) two times the Burgers vector magnitude. Atoms in (a) are colored by their potential energy. The pattern is symmetric with respect to the dashed mirror lines in (a). In (b) and (c), dislocation line segments are superimposed on the unrelaxed dichromatic pattern of the grain boundary.

Similar to the atomistic structure, the network predicted by the ROM-shown in Fig. (4.b) and (4.c)-also consists of predominantly screw character dis- 
location segments with $\frac{1}{2}\langle 111\rangle$ - and $\langle 100\rangle$-type Burgers vectors, the former with approximately twice the length of the latter. However, unlike in the atomistic structure, for a core cutoff radius of one quarter of the Burgers vector, the ROM network consists of slightly distorted hexagons with no lines of mirror symmetry, as evidenced by the unequal values of the angles $\alpha_{1}$ and $\alpha_{2}$ between the short and long dislocation segments in Fig. (4.b). In addition to the geometry shown in Fig. (4.b), the ROM also predicts another stable dislocation network configuration with identical energy by reversing the circulation of the Burgers vectors and with the values of the two angles $\alpha_{1}$ and $\alpha_{2}$ reversed. Thus, the complete elastic energy landscape of the $\mathrm{Nb}$ t-GB dislocation network with this cutoff radius-expressed in terms of the nodal positions-shares the same symmetry as the GB dichromatic pattern itself, but the individual dislocation configurations corresponding to the minima in that landscape do not.

We have analyzed the discrepancy between the ROM and atomistic structures in detail and confirmed that, for core cutoff radii of one quarter of the Burgers vector, it occurs systematically for all the twist angles we investigated and is not due to inadequate relaxation of either model. Its cause ultimately traces back to the character dependence of dislocation strain energies in bcc crystals $[51,52]$. In elastically isotropic bcc crystals, screw dislocations have the lowest energy per unit length. By contrast, in elastically anisotropic materials, the dislocation energy per unit length is lowest for mixed dislocations. For instance, in Nb, dislocation arrays with [111]-type Burgers vectors exhibit a deviation of $\sim 10^{\circ}$ with respect to perfect screw character [10]. The asymmetry of the distorted hexagons in Fig. (4.b) in- 
creases the edge component of the constituent dislocation segments, thereby reducing the elastic strain energy, as compared to the perfectly symmetric hexagons in Fig. (4.a).

Interestingly, when the core cutoff radius is increased to two times the Burger vector, the relaxed dislocation network predicted by the ROM is symmetric, as shown in Fig. (4.c). At first, it might be tempting to say that using a larger core cutoff changes the character dependence of the dislocation elastic energy, e.g. by lowering the energy of the pure screw relative to a mixed character. However, the form of the elastic field around an isolated dislocation has no characteristic length scale, so changing the core cutoff cannot lead to any change in the character dependence of dislocation properties [53]. Rather, the difference between the patterns in Fig. (4.b) and Fig. (4.c) is likely due to the length scale of the GB dislocation network itself, in particular to features of its elastic field within a distance of $\sim 2 b$ from the three-fold junctions that, when excluded from the elastic energy calculation, shift the elastic energy minimum to the symmetric state. Evidence for such near-node effects at twist grain boundaries along $\{110\}$-type planes in bec metals has been found in phase field simulations of dislocation networks, where dislocations are seen to acquire a slight curvature near three-fold junctions in some materials [54].

\section{2. $\mathrm{Ag} / \mathrm{V}$ interfaces}

Figure (5) plots energies of $\mathrm{Ag} / \mathrm{V}$ interfaces as a function of twist angle, $\theta$. As discussed in Ref. [11], the energies of heterophase interfaces, such as $\mathrm{Ag} / \mathrm{V}$, may be viewed as the sum of a chemical contribution, which is due to the difference in bonding between the two elements in the coherent 
reference state, and a contribution from the misfit dislocation network, which is associated with the relaxation of coherency. Only the latter depends on the twist angle while the former is a constant, independent of $\theta$. Our ROM only computes the elastic contribution to the misfit dislocation network energy. Thus, to ease comparison of energies computed from atomistics to those computed using the ROM, we have shifted all plots in Fig. (5) so that their minima are at an energy value of zero. For the atomistic calculations, a downward shift of $0.85 \mathrm{~J} / \mathrm{m}^{2}$ was imposed while all the ROM calculations were shifted downward by $0.24 \mathrm{~J} / \mathrm{m}^{2}$. The difference between these shift values, i.e. $0.61 \mathrm{~J} / \mathrm{m}^{2}$, is due to the chemical bonding contribution to the total interface energy. It is substantially larger than the elastic contribution. This conclusion is consistent with previous first-principles calculations, such as the one on Fe/VN interfaces reported by Johansson et al. in Ref. [55].

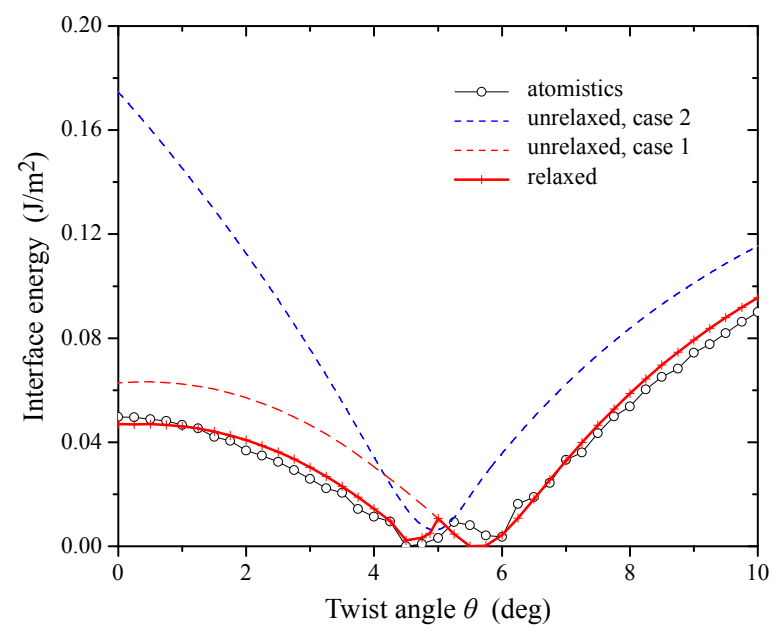

Figure 5: $\mathrm{Ag} / \mathrm{V}$ interface energies computed as a function of $\theta$ using our dislocation-based $\mathrm{ROM}$ and atomistic modeling.

The Ag/V interface energies computed using the atomistic model exhibit 
local maxima at $\theta=0^{\circ}$ (the Nishiyama-Wasserman orientation relation) and $\theta=5.25^{\circ}$ (the Kurdjumov-Sachs orientation relation), two nearly degenerate minima at $\theta=4.5^{\circ}$ and $\theta=6^{\circ}$, and a monotonically increasing energy for twist angles greater than $6^{\circ}$. Fig. (5) plots ROM energies for two unrelaxed dislocation network configurations, labeled "case 1" and "case 2". These cases correspond to two different solutions to the qFBE obtained by selecting two different combinations of misfit dislocation Burgers vectors, following the terminology introduced in Ref. [11]. In that paper, we defined case 1 as the solution obtained using Burgers vectors $\boldsymbol{b}_{1}$ and $\boldsymbol{b}_{2}$ and case 2 as that obtained using $\boldsymbol{b}_{1}$ and $\boldsymbol{b}_{3}$, with $\boldsymbol{b}_{1}=a_{\mathrm{ref}} / 2[\overline{1} 01], \boldsymbol{b}_{2}=a_{\mathrm{ref}} / 2[0 \overline{1} 1]$, and $\boldsymbol{b}_{3}=a_{\mathrm{ref}} / 2[\overline{1} 10]$ in the reference crystal. Case 1 has the lower energy for all twist angles, except in the interval $\sim 4.25^{\circ}<\theta<\sim 5.25^{\circ}$, where the energy of the latter is lower. One point of intersection between the case 1 and case 2 plots in Fig. (5) occurs near a local energy maximum, close the Kurdjumov-Sachs orientation relation. Each of the unrelaxed configurations predicts one energy minimum, with twist angles and energies in reasonable agreement with one of the minima in the atomistic model. Moreover, the energies of case 1 are in quantitative agreement with the atomistic model for $\theta>6^{\circ}$. However, at lower twist angles $\left(\theta<\sim 6^{\circ}\right)$, case 1 systematically overpredicts the interface energy by approximately $20 \mathrm{~mJ} / \mathrm{m}^{2}$.

As demonstrated in Fig. (5), relaxation of the dislocation network structure in the ROM removes nearly all discrepancies in energy between the two models. Quantitatively accurate predictions of interface energies are achieved over the full range of twist angles, with the greatest differences being on the order of $10 \mathrm{~mJ} / \mathrm{m}^{2}$ and occurring within a relatively narrow range of twist 
angles centered approximately on $\theta=5.5^{\circ}$. Most notably, the match in the energy and twist angle of the minimum near $\theta=4.5^{\circ}$ improves and the discrepancy between the ROM and atomistic energies for $\theta<\sim 4^{\circ}$ is removed.

The dislocation network geometries predicted by atomistics and the ROM are compared in Fig. (6) for several representative twist angles. All these examples exhibit good qualitative agreement between the two modeling methods, with the general shape of the relaxed ROM patterns matching that of the atomistic models. Small quantitative discrepancies in the lengths and angles of individual dislocations are nevertheless apparent, e.g., in the orientation of the dislocation segments colored blue for $\theta=2^{\circ}$ in Fig. (6.b). Interestingly, the ROM predicts no dissociation of four-fold dislocation nodes for $\theta=8^{\circ}$, thereby explaining why the unrelaxed network labeled "case 1" in Fig. (5) predicts the energy of this interface so accurately.

\section{Discussion}

We have investigated the effect of dislocation network relaxation on the accuracy of a dislocation-based reduced order model (ROM) of interface structure. Two types of model interfaces were investigated: pure twist grain boundaries on $\{110\}$-type planes in Nb (Nb t-GBs) and heterophase interfaces between a $\{111\}$ plane in $\mathrm{Ag}$ and a $\{110\}$ plane in $\mathrm{V}$ ( $\mathrm{Ag} / \mathrm{V}$ interfaces). The energies and dislocation network structures predicted by the ROM were compared to corresponding atomistic simulations for a range of twist angles.

We find that incorporating relaxation leads to improved predictions of interface energies for $\mathrm{Ag} / \mathrm{V}$ interfaces, yielding nearly perfect quantitative agreement with atomistic models. In particular, it did not appear that these predictions might be improved by incorporating a dislocation core model 
(a) NW OR, i.e. $\theta=0^{\circ}$

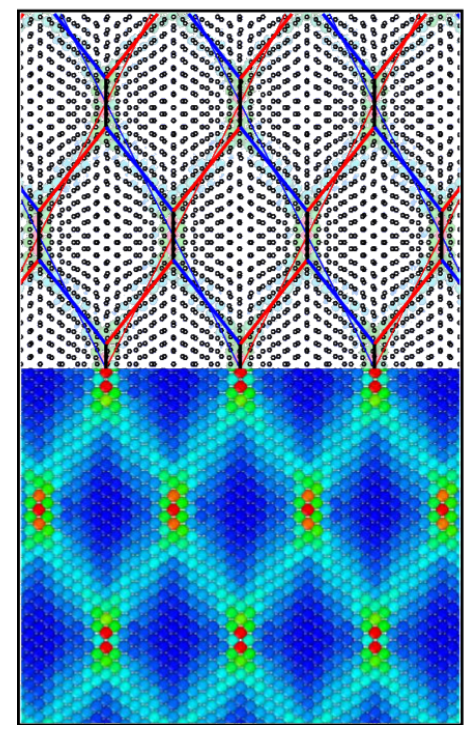

(b) $\theta=2^{\circ}$

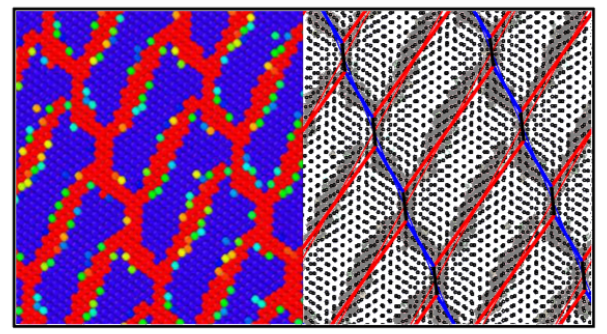

(c) $\theta=3^{\circ}$

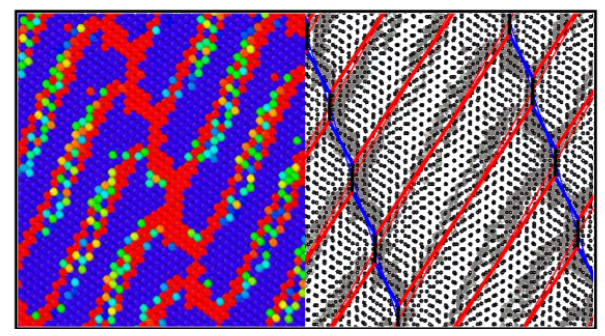

(d) $\theta=8^{\circ}$

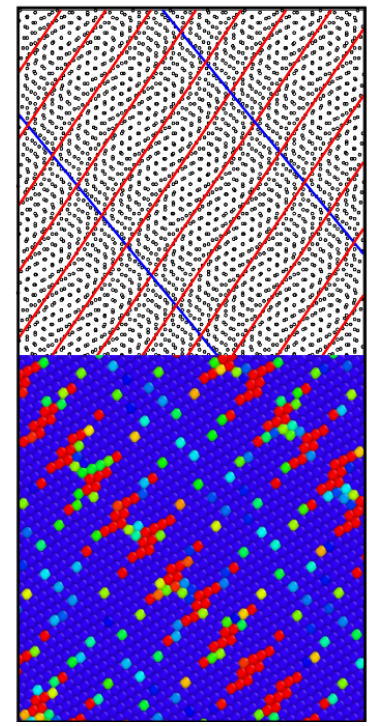

Figure 6: The dislocation structures of the $\mathrm{Ag} / \mathrm{V}$ interface obtained by the atomistic modeling (atoms coloured by their local potential energies using the same color bar as in Fig. (4) from $-5.13 \mathrm{ev}$ (blue) to $-5.11 \mathrm{ev}$ (red) and dislocation-based ROM (dislocation segments superimposed on dichromatic patterns). In (a), (b), and (c), the black line segment is the dislocation segment created upon dissociation of a four-fold node into two three-fold nodes. There is no such segment in (d) because the four-fold node in this network does not dissociate. The thin black lines in (a) illustrate the shape of the initial, unrelaxed dislocation network.

into our ROM. This apparent insensitivity to core structure may be due to the relatively large (1 nm-scale [11]) width of misfit dislocation cores in such interfaces as well as their confinement to the interface plane [20]. It indicates that the effectiveness of linear elastic, dislocation-based models in predicting interface energies and structures is greater than anticipated for some heterophase interfaces, such as the $\mathrm{Ag} / \mathrm{V}$ interface studied here.

Our investigation also shows that, while incorporating relaxations improved quantitative predictions of $\mathrm{Ag} / \mathrm{V}$ interface energies, it did not alter the qualitative features of the energy vs. twist angle plots obtained from un- 
relaxed dislocation models. In particular, it appears that qualitative features of this curve-such as the number of energy minima and maxima as well as their twist angles and relative energies-may be obtained via consideration of unrelaxed structures alone. Relaxation of the dislocation network is not essential for predicting those aspects of the interface energy dependence on twist angle. However, dislocation network relaxation is essential for correctly predicting the geometry of the dislocation networks in these interfaces.

For $\mathrm{Nb}$ t-GBs, we observed marked differences between energies computed using our ROM and atomistic models. We attribute these differences to the significant contribution of dislocation cores to the total energy of these interfaces: a contribution naturally accounted for in the atomistic model, but not in our ROM. In particular, the incorporation of dislocation network relaxations in the ROM does not resolve the observed discrepancies. Moreover, use of short $(\sim b / 4)$ core cutoff radii leads to asymmetric lowest energy network geometries, contrary to atomistic models. Thus, to better predict the energies of $\mathrm{Nb}$ t-GBs, the ROM should be augmented with a core model. One approach might be to include a Peierls-Nabarro type calculation in the ROM with gamma-surfaces obtained from first principles calculations [35]. Some phase field models of dislocation behavior already use such techniques to model dislocation core spreading as well as the corresponding core energy $[41,54]$.

Additional improvements to our ROM will further broaden its applicability to a wider range of interfaces. For example, incorporating dissociation of dislocations into partials would enable us to simulate the structure of interfaces such as twist grain boundaries and heterophase interfaces between 
$\{111\}$ facets in FCC solids [56]. Another challenge is the incorporation of dislocations with a third, linearly independent Burgers vector [33]. This improvement would allow us to apply the ROM to grain boundaries and fcc/bcc interfaces of any crystallographic character. However, lack of translational symmetry in the dislocation networks found in such general interfaces presents a significant obstacle for the present formulation of our ROM, which is based on Fourier series expansions of the interface elastic fields.

Certain temperature-related effects may easily be incorporated into our ROM. For example, under isothermal conditions, the temperature dependence of both the elastic constants and lattice parameters may be taken into account simply by substituting into the model the values at the temperature of interest. However, spatially varying temperature distributions generate non-uniform thermal stresses that may interact with dislocation stress fields in a more complex manner and alter the relaxed dislocation patterns. Moreover, temperature-dependent mobility relations at three- and four-fold dislocation nodes may be used to model dislocation network as a function of time, as commonly done in standard dislocation dynamics simulations [57].

Finally, there is one point of divergence between the ROM and atomistic models that may be inevitable: while dislocation networks modeled by the ROM are perfectly periodic, atomistic models can only exhibit perfect periodicity for ideal coincident site lattice (CSL) boundaries [16]. Misfit dislocation networks in general interfaces-including general grain boundaries-cannot be perfectly periodic. Rather, all unit cells in their dislocation patterns are slightly different, as the dislocation cores in the cells adjust to the positions of the discrete atoms making up the interface. The absence of atomic degrees 
of freedom may make it impossible for the ROM to capture such small-scale, cell-to-cell variations in the shape of the dislocation network.

\section{References}

[1] A. Misra, J.P. Hirth, and H. Kung, Single-dislocation-based strengthening mechanisms in nanoscale metallic multilayers, Philos. Mag. A 82, 29352951 (2002).

[2] I.J. Beyerlein, M.J. Demkowicz, A. Misra, and B.P. Uberuaga, Defectinterface interactions, Prog. Mater. Sci. 74, 125-210 (2015).

[3] A. Kashinath, A. Misra, and M.J. Demkowicz. Stable storage of helium in nanoscale platelets at semicoherent interfaces, Phys. Rev. Lett. 110, 086101 (2013).

[4] M.J. Demkowicz, A. Misra, and A. Caro. The role of interface structure in controlling high helium concentrations, Curr. Opin. Solid State Mat. Sci. 16, 101-108 (2012).

[5] W.S. Yu, and M.J. Demkowicz. Non-coherent $\mathrm{Cu}$ grain boundaries driven by continuous vacancy loading, J. Mater. Sci. 50, 4047-4065 (2015).

[6] S. Mao, S. Shu, J. Zhou, R.S. Averback, and S.J. Dillon. Quantitative comparison of sink efficiency of $\mathrm{Cu}-\mathrm{Nb}, \mathrm{Cu}-\mathrm{V}$ and $\mathrm{Cu}-\mathrm{Ni}$ interfaces for point defects, Acta Mater. 82, 328-335 (2015).

[7] B.P. Uberuaga, L.J. Vernon, E. Martinez, and A.F. Voter. The relationship between grain boundary structure, defect mobility, and grain boundary sink efficiency, Scientific Reports 5, 9095 (2014). 
[8] Y. Mishin, M. Asta, and J. Li. Atomistic modeling of interfaces and their impact on microstructure and properties, Acta Mater. 58, 11171151 (2010).

[9] A. Vattré. Elastic strain relaxation in interfacial dislocation patterns: I. A parametric energy-based framework, J. Mech. Phys. Solids 105, 254-282 (2017).

[10] A. Vattré. Elastic strain relaxation in interfacial dislocation patterns: II. From long- and short-range interactions to local reactions, J. Mech. Phys. Solids 105, 283-305 (2017).

[11] A.J. Vattré, N. Abdolrahim, K. Kolluri, and M.J. Demkowicz. Computational design of patterned interfaces using reduced order models, Scientific Reports 4, 6231 (2014).

[12] R.C. Pond, and D.S. Vlachavas. Bicrystallography, Proc. Royal Soc. A 386, 95-143 (1983).

[13] W. Bollmann. Crystal defects and crystalline interfaces, Springer-Verlag, Berlin (1970).

[14] B. Runnels, I.J. Beyerlein, S. Conti, and M. Ortiz. An analytical model of interfacial energy based on a lattice-matching interatomic energy, J. Mech. Phys. Solids 89, 174-193 (2016).

[15] J.P. Hirth, R.C. Pond, R.G. Hoagland, X.Y. Liu, and J. Wang. Interface defects, reference spaces and the Frank-Bilby equation, Prog. Mater. Sci. 58, 749-823 (2013). 
[16] A.P. Sutton, and R.W. Balluffi. Interfaces in Crystalline Materials, Oxford University Press, Oxford (1995).

[17] J.B. Yang, Y. Nagai, Z.G. Yang, and M. Hasegawa. Quantization of the Frank-Bilby equation for misfit dislocation arrays in interfaces, Acta Mater. 57, 4874-4881 (2009).

[18] A. Sangghaleh, and M.J. Demkowicz. AIDA: A tool for exhaustive enumeration of solutions to the quantized Frank-Bilby equation, Comput. Mater. Sci. 145, 35-47 (2018).

[19] J. Han, V. Vitek, and D.J. Srolovitz. The grain-boundary structural unit model redux, Acta Mater. 133, 186-199 (2017).

[20] M.J. Demkowicz, J. Wang, and R.G. Hoagland. Interfaces between dissimilar crystalline solids. in: Hirth JP, (Ed.). Dislocations in Solids, Elsevier, Amsterdam, 14, 141-207 (2008).

[21] W.T. Read, and W. Shockley. Dislocation models of crystal grain boundaries, Phys. Rev. 78, 275-289 (1950).

[22] A.J. Vattré, T. Jourdan, H. Ding, M.C. Marinica, and M.J. Demkowicz. Non-random walk diffusion enhances the sink strength of semicoherent interfaces, Nat. Commun. 7, 10424 (2016).

[23] Y.J. Gu, J. Han, S.Y. Dai, Y.C. Zhu, Y. Xiang, and D.J. Srolovitz. Point defect sink efficiency of low-angle tilt grain boundaries, J. Mech. Phys. Solids 101, 166-179 (2017). 
[24] R.F. Zhang, T.C. Germann, J. Wang, X.Y. Liu, and I.J. Beyerlein. Role of interface structure on the plastic response of $\mathrm{Cu} / \mathrm{Nb}$ nanolaminates under shock compression: Non-equilibrium molecular dynamics simulations, Scr. Mater. 68, 114-117 (2013).

[25] R.F. Zhang, J. Wang, I.J. Beyerlein, and T.C. Germann. Dislocation nucleation mechanisms from fcc/bcc incoherent interfaces, Scr. Mater. 65, 1022-1025 (2011).

[26] M.J. Demkowicz, and L. Thilly. Structure, shear resistance, and interaction with point defects of interfaces in $\mathrm{Cu}-\mathrm{Nb}$ nanocomposites synthesized by severe plastic deformation, Acta Mater. 59, 7744 (2011).

[27] A.J. Vattré, and M.J. Demkowicz. Determining the Burgers vectors and elastic strain energies of interface dislocation arrays using anisotropic elasticity theory, Acta Mater. 61, 5172-5187 (2013).

[28] A.J. Vattré, and M.J. Demkowicz. Partitioning of elastic distortions at a semicoherent heterophase interface between anisotropic crystals, Acta Mater. 82, 234-243 (2015).

[29] A.J. Vattré, and M.J. Demkowicz. Effect of interface dislocation Burgers vectors on elastic fields in anisotropic bicrystals, Comput. Mater. Sci. 88, 110-115 (2014).

[30] R. Madec, B. Devincre, and L.P. Kubin. On the nature of attractive dislocation crossed states, Comput. Mater. Sci. 23, 219-224 (2002). 
[31] C.S. Hong, X.X. Huang, and G. Winther. Dislocation content of geometrically necessary boundaries aligned with slip planes in rolled aluminium, Philos. Mag. 93, 3118-3141 (2013).

[32] S. Amelinckx. The direct observation of dislocations, Academic Press, New York (1964).

[33] N. Abdolrahim, and M.J. Demkowicz. Determining coherent reference states of general semicoherent interfaces, Comput. Mater. Sci. 118, 297308 (2016).

[34] S.Y. Dai, Y. Xiang, and D.J. Srolovitz. Structure and energy of (111) low-angle twist boundaries in $\mathrm{Al}, \mathrm{Cu}$ and Ni, Acta Mater. 61, 1327-1337 (2013).

[35] S.Y. Dai, Y. Xiang, and D.J. Srolovitz. Atomistic, generalized PeierlsNabarro and analytical models for (111) twist boundaries in $\mathrm{Al}, \mathrm{Cu}$ and Ni for all twist angles, Acta Mater. 69, 162-174 (2014).

[36] Z. Nishiyama. Mechanism of transformation from face-centred to bodycentred cubic lattice, Science Reports of the Tohoku Imperial University 23, 637-664 (1934).

[37] G. Wasserman. Arch. Eisenhuttenwesen 16, 647 (1933).

[38] Q.M. Wei, and A. Misra. Transmission electron microscopy study of the microstructure and crystallographic orientation relationships in $\mathrm{V} / \mathrm{Ag}$ multilayers, Acta Mater. 58 4871-4882 (2010). 
[39] X.Y. Liu, R.G. Hoagland, M.J. Demkowicz, M. Nastasi, and A. Misra. The influence of lattice misfit on the atomic structures and defect energetics of face centered cubic-body centered cubic interfaces, J. Eng. Mater. Technol. 134, 021012 (2012).

[40] Z.H. Liu, Y.X. Feng, and J.X. Shang. Characterizing twist grain boundaries in BCC Nb by molecular simulation: Structure and shear deformation, Appl. Surf. Sci. 370, 19-24 (2016).

[41] D. Qiu, P. Zhao, C. Shen, W. Lu, D. Zhang, M. Mrovec, and Y. Wang. Predicting grain boundary structure and energy in BCC metals by integrated atomistic and phase-field modeling, Acta Mater., in press (2018).

[42] M.S. Daw, and M.I. Baskes. Embedded-atom method - derivation and application to impurities, surfaces, and other defects in metals, Phys. Rev. B 29, 6443-6453 (1984).

[43] L. Zhang, E. Martinez, A. Caro, X.Y. Liu, and M.J. Demkowicz. Liquid-phase thermodynamics and structures in the $\mathrm{Cu}-\mathrm{Nb}$ binary system, Model. Simul. Mater. Sci. Eng. 2, 025005 (2013).

[44] Q.M. Wei, X.Y. Liu, A. Misra. Observation of continuous and reversible bcc-fcc phase transformation in Ag/V multilayers, Appl. Phys. Lett. 98, 111907 (2011).

[45] A.R. Leach. Molecular Modelling: Principles and applications. 2nd ed., Prentice Hall, Harlow, England (2001).

[46] S. Plimpton. Fast parallel algorithms for short-range moleculardynamics, J. Comput. Phys. 117, 1-19 (1995). 
[47] J. Li. AtomEye: an efficient atomistic configuration viewer, Model. Simul. Mater. Sci. Eng. 11, 173-177 (2003).

[48] A.N. Stroh. Dislocations and cracks in anisotropic elasticity, Philos. Mag. 3, 625-646 (1958).

[49] A.N. Stroh. Steady state problems in anisotropic elasticity, J. Math. Phys. 41, 77-103 (1962).

[50] J.B. Yang, Y. Nagai, M. Hasegawa, and Y.N. Osetsky. Atomic scale modeling of $\{110\}$ twist grain boundaries in alpha-iron: Structure and energy properties, Philos. Mag. 90, 991-1000 (2010).

[51] D.M. Barnett, R.J. Asaro, S.D. Gavazza, D.J. Bacon, and R.O. Scattergood. The effects of elastic anisotropy on dislocation line tension in metals, J. Phys. F: Metal Phys. 2, 854-864 (1972).

[52] R.J. Asaro, and J.P. Hirth, Planar dislocation interactions in anisotropic media with applications to nodes, J. Phys. F: Metal Phys. 3, 1659-1671 (1973).

[53] J.P. Hirth, and J. Lothe. Theory of Dislocations. 2nd ed., Wiley, New York (1982).

[54] Y.Z. Wang, and J. Li. Phase field modeling of defects and deformation, Acta Mater. 58, 1212-1235 (2010).

[55] S.A.E. Johansson, M. Christensen, and G. Wahnström. Interface energy of semicoherent metal-ceramic Interfaces, Phys. Rev. Lett. 95, 226108 (2005). 
[56] S. Shao, J. Wang, A. Misra, R.G. Hoagland. Spiral patterns of dislocations at nodes in (111) semi-coherent FCC interfaces, Scientific reports 3, 2448 (2013).

[57] L. Kubin. Dislocations, Mesoscale Simulations and Plastic Flow, Oxford University Press, Oxford, (2013). 